\title{
Study of the Interference Rejection Based on ATF in DSSS
}

\author{
Pinghua Zhang ${ }^{1,2, a}$, Hongli $\mathrm{Xu}^{1}{ }^{\text {,Manman Peng }}{ }^{2}$ and Luyun cao ${ }^{1}$ \\ ${ }^{1}$ Hunan Information College, School of Electronic Engineering, 410200 Changsha, China \\ ${ }^{2}$ Hunan University, College of Information Science and Engineering, 410082 Changsha, China
}

\begin{abstract}
A novel, adaptive time-frequency interference rejection for suppressing the interference in Direct Sequence Spread Spectrum communication systems is studied in this paper. For a time-localized interference, excision is performed in the time domain. When a frequency domain excision is decided, an adaptive sub-band transform is employed. The most proper sub-spectral split is defined according to the spectrum of the received signal. This joint treatment of time and frequency domains overcomes the performance limitations of the fixed transform-based excision algorithms. The paper presents the performance evaluation of the rejection technique via the analytical and computer simulation tools. It is found that the analytical performance tools match up with the computer simulation results. It is also shown that the proposed adaptive time-frequency interference rejection consistently outperforms the existing interference excision techniques in the literature.
\end{abstract}

\section{Introduction}

Spread spectrum technology has strong anti-interference ability and multiple access capability, and can timing and ranging precisely. So it is widely used in military and civil communication systems. At the same time, the research on the communication system of the spread spectrum communication system has become a hot spot in the research of reconnaissance, jamming and antijamming. Because the mechanism of spread spectrum technology is to use a relatively wide frequency band, so it is more easily to be subject to the attacks of single narrowband interference, multiple narrowband interference, broadband interference and other interferences. In many cases, the cost is too high to enhance the ability of resisting interference by improving the system's processing gain. Sometimes it is even impossible to achieve. So we need to use effective signal processing technology to suppress the interference, to ensure the accurate transmission and reception of information ${ }^{[1]}$.

One of the most useful techniques in the antijamming technology is the transform domain filtering processing technique. Existing transform domain filters adopt the frequency domain processing technology using fixed transformation base, such as fast Fourier transform (FFT), the signal is mapped into the frequency domain, but the interference suppression performance is limited by the size and accuracy of FFT, especially when the interference is leaked into the adjacent bands due to the sub-band spectrum which like $\sin (x) / x$, and also when the narrowband noise threshold is difficult to determine as the background additive Gaussian noise is too strong. There is also no need to decompose the disturbed frequency band and the free frequency band to the same resolution ${ }^{[2]}$. Later, sub-band transform interference suppression is put forward in spread spectrum communication systems, although it has a better frequency domain energy aggregation, but has the same limitations with the fixed transform base frequency domain interference processing technology, such as spectrum leakage between adjacent sub bands and fixed time-frequency resolution. Especially for time varying and non-stationary interferences, such as pulse jamming, the transform domain processing technique is invalid ${ }^{[3]}$.

In this paper, a new method of anti-jamming in the transform domain is studied--adaptive time-frequency interference rejection (ATF). The proposed algorithm can generate a sub-band decomposition tree with different levels due to different input signal. In the process of the sub-band decomposition, when the intensity of the transform domain energy exceeds the time domain or a given threshold, the node will continue to decompose. Therefore, the algorithm avoids unnecessary decomposition, and can greatly reduce the calculation of addition and multiplication. Compared with a fixed structure filter, one of the biggest advantages of ATF is that it can adaptively change the hierarchical structure of the sub band filter banks, reduce the segmentation of the transform domain, and can locate the interference signal of the frequency domain more accurately. Thus it can reduce the sensitivity of interference, and it is a robust technique for suppressing interference. At the same time, the adopted adaptive sub tree structure algorithm can overcome the shortcomings of fixed spectral resolution in the existing transform domain processing techniques ${ }^{[4]}$.

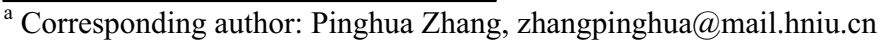




\section{Direct Spread Spectrum System}

Take the most common way - direct spread spectrum system (DSSS) for example, the input signal of the adaptive time-frequency (ATF) interference suppressor is ${ }^{[5]}$

$$
r(k)=d_{b} c(k)+n(k)+j(k)
$$

In the formula, the value of $d_{b}$ is \pm 1 equiprobability distributed; $c(k)$ is the spreading sequences, the system processing gain is $L ; n(k)$ is the additive white Gaussian noise of zero mean and variance $D, j(k)$ is the interference signal of mean zero and covariance $R_{j}(k)$.

\section{Adaptive Time-frequency interference Rejection ATF}

The main idea of the interference suppression technology studied in the paper is to check the received signal $r(k)$ is suitable or not for interference suppression in the time domain. If yes, there is no need for frequency domain processing. When it is determined to eliminate the interference in frequency domain, the adaptive sub band transformation technology (also known as adaptive wavelet packet Technology), the signal is decomposed according to the frequency domain characteristics of the received signal. In order to find the optimal wavelet packet tree structure to reduce the frequency of interference, we use the energy aggregation degree to reflect the distribution of the signal power spectrum density. The energy aggregation degree of M-band is defined as

$$
G=\frac{\delta_{x}^{2}}{\left[\prod_{i=1}^{M} \delta_{i}^{2}\right]^{1 / M}}
$$

Where $\delta_{x}^{2}$ is the variance of input signal, $\delta_{i}^{2}$ is the output variance of the $i$-th sub-band. The bigger $G$ is, the less flat the frequency spectrum of the output signal is, and the signal energy is more focused on some sub bands [6].

The concrete steps of the adaptive tree structure algorithm are described as follows:

1) Calculate the power spectrum density of the received signal $P_{x x}(\omega)$.

2) a. Calculate the sub-band variances $\delta_{2 l}^{2}$ and $\delta_{2 h}^{2}$ of the two sub-band decomposition and the corresponding energy accumulation degree $G(2)$.

b. Calculate the sub-band variances $\delta_{3 l}^{2}, \delta_{3 b}^{2}$ and $\delta_{3 h}^{2}$ of the three sub-band decomposition and the corresponding energy accumulation degree $G(3)$.

3 ) If $G(2) \leqslant T$ and $G(3) \leqslant T$, means that the signal spectrum is flat, and the decomposition will stop ( $T$ is the pre-defined threshold).

Otherwise, if $G(2) \geqslant G(3)$, two band decomposition is used, and if $G(2)<G(3)$, then three band decomposition is used.
4) Repeat step 2) and step 3) until there is no node can be decomposed.

\section{Performance Analysis}

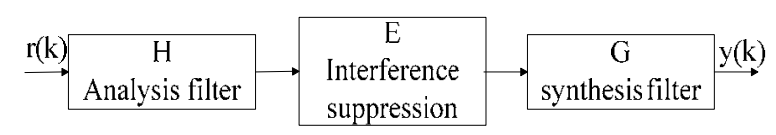

Figure 1. Diagram of interference suppression

Let $\left\{h_{i}(n)\right\}$ and $\left\{g_{i}(n)\right\}$ be the analysis and synthesis filter function, the coefficient $\left\{e_{i}\right\}$ is the interference weight, the sub-band with interference is multiplied by 0 , the sub band without interference is multiplied by 1 . Then the output of the sub-band can be written as

$$
y_{i}(k)=\left[e_{i}(k) * h_{i}(k) * g_{i}(k)\right] * r(k)
$$

The equivalent filter of the sub-band is recorded as

$$
\begin{aligned}
t_{i}(k) & @ e_{i}(k) * h_{i}(k) * g_{i}(k) \\
& =e_{i}(k) * \sum_{m=0}^{N-1} h_{i}(m) g_{i}(k-m)
\end{aligned}
$$

Since $g_{i}(k)$ is the time reversal of $h_{i}(k), t_{i}(k)$ is a linear phase FIR filter with a length $(2 N-1)$. For the contaminated sub-band

$$
e_{i}(k)=0 \rightarrow t_{i}(k)=0
$$

And the output signal is

$$
y(k)=\sum_{i=1}^{M} \sum_{l=0}^{2 N-2} t_{i}(l) r(k-l)
$$

The output variable $\varepsilon$ can be written as

$$
\begin{aligned}
\varepsilon & =\sum_{k=N}^{N+L-1} y(k) c(k) \\
& =\sum_{k=N}^{N+L-1}\left[\sum_{i=1}^{M} \sum_{l=0}^{2 N-2} t_{i}(l) r(k-l)\right] c(k)
\end{aligned}
$$

Then the mean of $\varepsilon\left(d_{b}= \pm 1\right.$ hypothetically) is

$$
E[\varepsilon]=L \sum_{i=1}^{M} t_{i}(0)-\frac{1}{L} \sum_{k=N}^{N+L-1} \sum_{i=1}^{M} \sum_{l=1}^{2 N-2} t_{i}(l)
$$


The variance of $\varepsilon$ is

$$
\begin{aligned}
& \operatorname{var}[\varepsilon]=L^{2} \sum_{i=1}^{M} \sum_{r=1}^{M} \sum_{l=0}^{2 N-2} t_{i}(l) t_{\mathrm{r}}(l) \\
& -\left[L \sum_{i=1}^{M} t_{i}(0)-\frac{1}{L} \sum_{k=N}^{N+L-1} \sum_{i=1}^{M} \sum_{l=1}^{2 N-2} t_{i}(l)\right]^{2} \\
& +L \sum_{i=1}^{M} \sum_{r=1}^{M} \sum_{l=0}^{2 N-2} \sum_{s=0}^{2 N-2} t_{i}(l) t_{r}(s) R_{j}(s-l) \\
& -\frac{1}{L} \sum_{k=N}^{N+L-1} \sum_{\substack{N+L=N \\
p \neq k}}^{M} \sum_{i=1}^{M} \sum_{r=1}^{M} \sum_{l=0}^{2 N-2} \\
& \sum_{s=0}^{2 N-2} t_{i}(l) t_{r}(s) R_{j}(k-l-p+s) \\
& +L \delta_{n}^{2} \sum_{i=1}^{M} \sum_{r=1}^{M} \sum_{l=0}^{2 N-2} t_{i}(l) t_{\mathrm{r}}(l) \\
& -\frac{1}{L} \sum_{k=N}^{N+L-1} \sum_{\substack{N=N \\
p \neq L-1}}^{M} \sum_{i=1}^{M} \sum_{r=1}^{M} \sum_{l=0}^{2 N-2} \\
& \sum_{s=0}^{2 N-2} t_{i}(l) t_{r}(s) \delta_{n}^{2} \delta(k-l-p+s)
\end{aligned}
$$

While

$$
E\left[\varepsilon^{2}\right]=E^{2}[\varepsilon]+\operatorname{var}[\varepsilon]
$$

The mean value of the decision variable is the useful signal, and the variance of the decision variable is the sum of the thermal noise, the residual interference component and the noise generated by the PN sequence itself, so the output signal to noise ratio (SNR) of the system is

$$
\operatorname{SIR}_{0} @ \frac{E^{2}[\varepsilon]}{\operatorname{var}[\varepsilon]}
$$

Then the bit error rate (BER) of the system is

$$
P_{e}=Q\left(\sqrt{S I R_{0}}\right)=Q\left(\sqrt{\frac{E^{2}[\varepsilon]}{\operatorname{var}[\varepsilon]}}\right)
$$

In the equal

$$
Q(x) @ \frac{1}{\sqrt{2 \pi}} \int_{x}^{\infty} e^{-y^{2} / 2} d y
$$

\section{Simulation Comparison \\ and \\ Performance}

In computer simulation, the DSSS system using length 63 PN code to spread spectrum, BPSK modulation, first consider the single tone jamming situation, when $j(k)=A \cos \left(\omega_{s} k+\theta\right)$. As $S I R=-20 d B, \omega_{s}=1.65$, the theoretical analysis and simulation comparison chart for bit error rate of ATF algorithm is shown in Figure 2. We can see that the theoretical analysis result is in agreement with the simulation result.

Figure 3 showing the bit error rate of the ideal case (there is no NBI), ATF algorithm and FFT algorithm respectively $\left(\omega_{s}=1.92\right)$. Results show that, the performance of the system using the ATF algorithm has been greatly improved.
Figure 4 shows the amplitude-frequency diagram of the corresponding unequal bandwidth adaptive tree structure in direct sequence spread spectrum disturbed by single tone interference. It shows the superiority of ATF algorithm in finding the best basement, which due to the use of an adaptive tree structure algorithm can be more flexible to choose the basement which matched with the center frequency of interference.

Then consider the Gaussian interference whose time domain energy is concentrated. Figure 5 shows the BER curve when the receiver is subject to time-varying broadband Gauss interference. The bit error rate performance of the ATF algorithm and FFT algorithm is shown respectively. In this case when $S I R=-20 \mathrm{~dB}$, the duty ratio of the interference is $10 \%$, the FFT algorithm obviously lost the interference suppression ability, but ATF algorithm still has good performance in interference suppression.

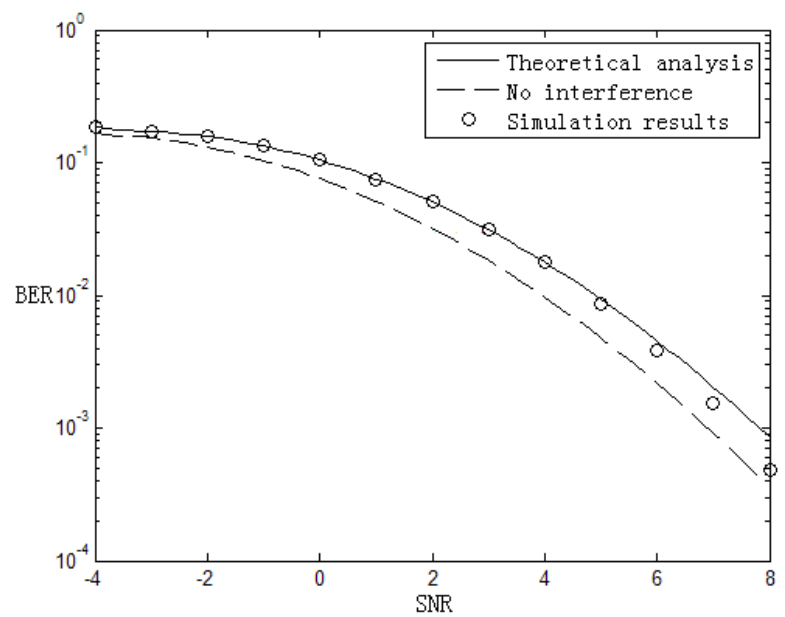

Figure 2. BER of theoretical analysis and simulation of ATF

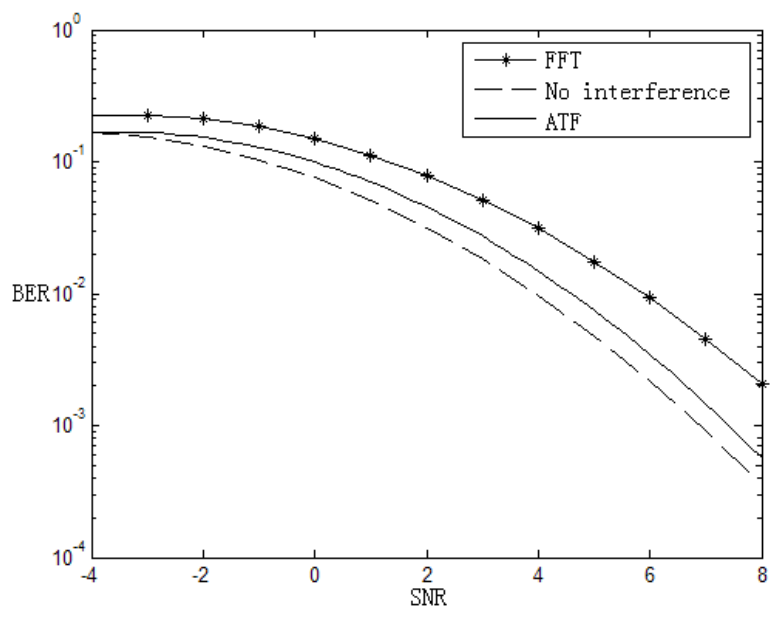

Figure 3. BER of single tone interference 


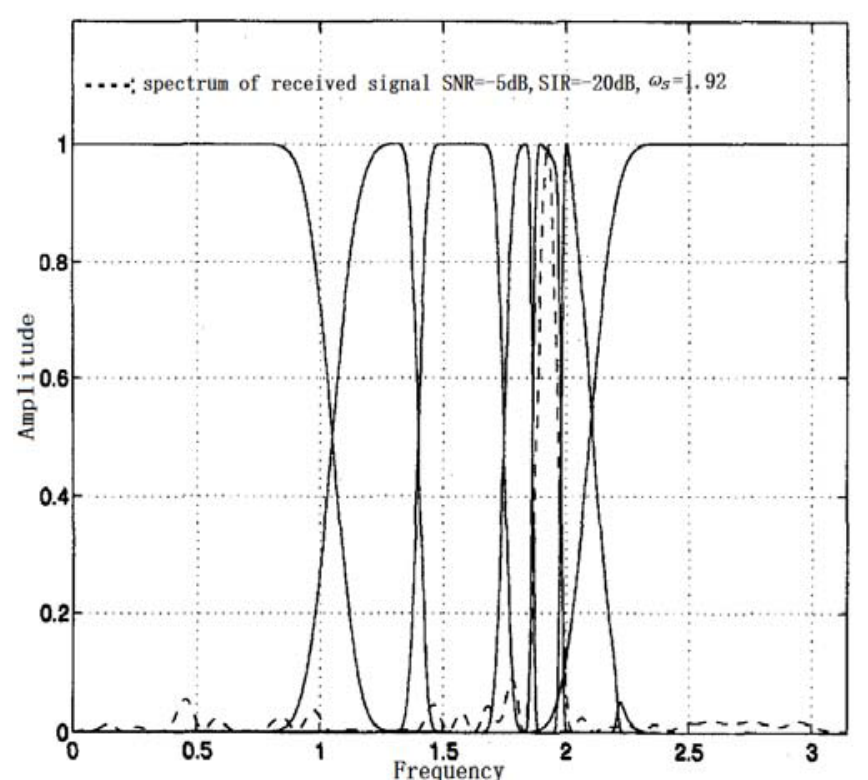

Figure 4. Structure of adaptive filter group disturbed by single tone interference

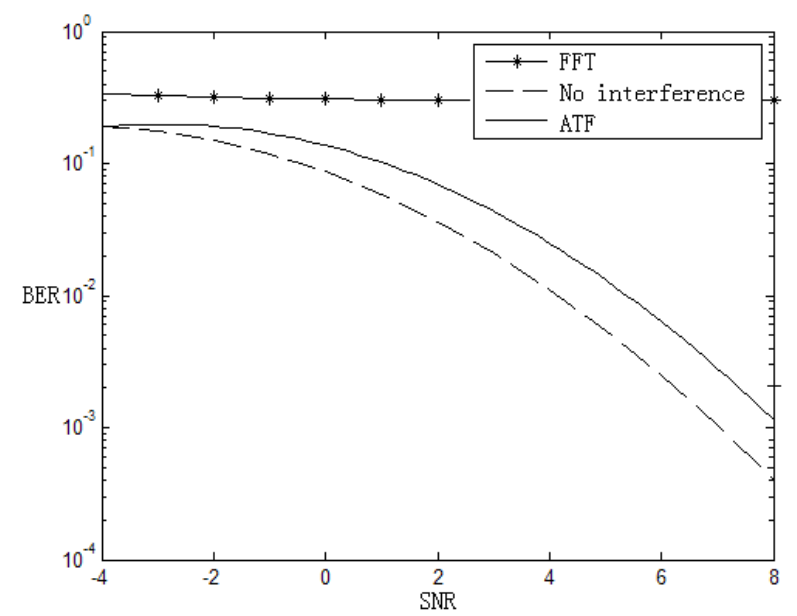

Figure 5. BER of time varying Gauss jamming

\section{Conclusion}

In this paper, a interference suppression technique based on adaptive time-frequency interference rejection is studied in the direct sequence spread spectrum communication system, the using of adaptive tree structure algorithm can be more flexible to select the basement matched with the center frequency of the interference. The closed form of bit error rate of the algorithm is derived. Theoretical analysis and computer simulation coincide. The results show that the algorithm is superior to the other frequency domain interference suppression techniques, and has a higher performance in bit error rate.

\section{Acknowledgements}

The scientific research project of Hunan Provincial Education Department (Number: 15C0982).
The Youth funded projects of Education Science "Twelfth Five Year Plan" in Hunan Province (Number: XJK015QZY007).

\section{References}

1. M.J.Medley,G.J.Saulnier,P.K.Da,IEEE Trans. on Commu. 45,11 (1997)

2. S.A.Wang, J.P. An, Signal Proc. Let. IEEE, 17,5 (2010)

3. C.Y. He, J. Guo, X.C Lu, X. Wang and F. Liu.CSNC2012, 147(2012)

4. M.V.Tazebay,A.N.Akansu,Speech and Signal Proc.Newark, 12(1995)

5. R.L.Peterson,R.E.Aieme,D.E.Borth,Introduction to Spread Spectrum Communication(Publishing House of Electronic Industry,Beijing,2006)

6. L.Ping,R.Lu,2013 Inter. Conf. on QRRMS Eng. ,2(2013) 